\title{
BMJ Open Immediate acupuncture with GB34 for biliary colic: protocol for a randomised controlled neuroimaging trial
}

\author{
Ning Sun (D) , , Dong-Mei He, ${ }^{2}$ Xiangyin Ye, ${ }^{1}$ Lei Bin, ${ }^{2}$ Yuanfang Zhou (D) , \\ Xiaodong Deng, ${ }^{1}$ Yuzhu Qu (D) , ${ }^{1}$ Zhengjie Li, ${ }^{1}$ Shirui Cheng (D) , ${ }^{1}$ Shuai Shao, ${ }^{2}$ \\ Feng-Juan Zhao, ${ }^{3}$ Tie-Huan Zhang, ${ }^{2}$ Jing Cai, ${ }^{4}$ Ruirui Sun (D) , ${ }^{1}$ Fan-rong Liang (D) ${ }^{1}$
}

To cite: Sun N, He D-M,

Ye X, et al. Immediate acupuncture with GB34 for biliary colic: protocol for a randomised controlled neuroimaging trial. BMJ Open 2022;12:e050413. doi:10.1136/ bmjopen-2021-050413

- Prepublication history for this paper is available online. To view these files, please visit the journal online (http://dx.doi. org/10.1136/bmjopen-2021050413).

Received 20 February 2021 Accepted 03 December 2021

Check for updates

(c) Author(s) (or their employer(s)) 2022. Re-use permitted under CC BY-NC. No commercial re-use. See rights and permissions. Published by BMJ.

${ }^{1}$ Acupuncture and Tuina School/ The 3rd Teaching Hospital, Chengdu University of Traditional Chinese Medicine, Chengdu,

Sichuan, China

${ }^{2}$ Emergency Department, The First People's Hospital of Longquanyi District, Chengdu, Sichuan, China

${ }^{3}$ Science and Education Department, The First People's Hospital of Longquanyi District, Chengdu, China

${ }^{4}$ Oncology-Blood Department, The First People's Hospital of Longquanyi District, Chengdu, China

Correspondence to

Dr Fan-rong Liang;

acuresearch@126.com

\section{ABSTRACT}

Introduction As the main manifestation of gallstone disease, biliary colic (BC) is an episodic attack that brings patients severe pain in the right upper abdominal quadrant. Although acupuncture has been documented with significance to lead to pain relief, the immediate analgesia of acupuncture for BC still needs to be verified, and the underlying mechanism has yet to be covered. Therefore, this trial aims first to verify the immediate painalleviation characteristic of acupuncture for $\mathrm{BC}$, then to explore its influence on the peripheral sensitised acupoint and central brain activity.

Methods and analysis This is a randomised controlled, paralleled clinical trial, with patients and outcome assessors blinded. Seventy-two patients with gallbladder stone disease presenting with $\mathrm{BC}$ will be randomised into a verum acupuncture group and the sham acupuncture group. Both groups will receive one session of immediate acupuncture treatment. Improvements in patients' BC will be evaluated by the Numeric Rating Scale, and the pain threshold of acupoints will also be detected before and after treatment. During treatment, brain neural activity will be monitored with functional near-infrared spectroscopy (fNIRS), and the needle sensation will be rated. Clinical and fNIRS data will be analysed, respectively, to validate the acupuncture effect, and correlation analysis will be conducted to investigate the relationship between pain relief and peripheral-cerebral functional changes.

Ethics and dissemination This trial has been approved by the institutional review boards and ethics committees of the First Teaching Hospital of Chengdu University of Traditional Chinese Medicine, with the ethical approval identifier $2019 \mathrm{KL}-029$, and the institutional review boards and ethics committees of the First People's Hospital of Longquanyi District, with the ethical approval identifier AFKY-2020071. The results of this trial will be disseminated through peer-reviewed publications and conference abstracts or posters.

Trial registration number CTR2000034432.

\section{INTRODUCTION}

Gallstone disease is one of the most common gastrointestinal and biliary system disorders. ${ }^{1}$ As the main manifestation of gallstone disease, biliary colic (BC) is severe pain in the right upper abdominal quadrant that
Strengths and limitations of this study

This randomised controlled trial will first use func tional near-infrared spectroscopy (fNIRS) to investigate cerebral changes during acupuncture for biliary colic (BC).

- Combining observations of both peripheral acupoint sensitisation and cerebral activity influenced by acupuncture will contribute to exploring the partial mechanism of acupuncture for $\mathrm{BC}$ alleviation.

- Both objective and subjective outcomes will be used to evaluate the effects of acupuncture treatment on $\mathrm{BC}$.

- Owing to a limited number of fNIRS measurement channels, we will restrict our analysis to the dorsolateral prefrontal cortex and primary somatosensory cortex of the patients.

- The cumulative effect of acupuncture for patients with gallstone disease with recurrent pain and accompanying discomforts, such as nausea and vomiting, will be further studied in the future.

attacks patients for at least $20-30 \mathrm{~min}^{2-4}$ In the developed world, $10 \%-15 \%$ of adults have gallstones, and $\mathrm{BC}$ occurs in $1 \%-4 \%$ of those with gallstones each year. ${ }^{5}$ It is reported that the cumulative probability of developing $\mathrm{BC}$ in initially asymptomatic people is approximately $11.9 \% \pm 3.0 \%$ at 2 years and $25.8 \% \pm 4.6 \%$ at 10 years. ${ }^{6}$ Moreover, BC can significantly impair sleep, mood and quality of life and cause major economic and social burdens. ${ }^{78}$

Compelling evidence indicates that nonsteroidal anti-inflammatory drugs (NSAIDs) are effective in relieving BC. ${ }^{9}$ However, long-term use of these medications has been considered to carry side effects. ${ }^{10-12}$ As one of the complementary therapies, acupuncture is thought to be effective for alleviating acute pain with rare side effects. ${ }^{13-15}$ For many diseases with visceral pain, acupuncture has been proven to be effective. For instance, Zhao et al demonstrated that acupuncture is 
beneficial for the alleviation of angina as an adjunctive therapy. ${ }^{16}$ More recently, Pei et al reported that acupuncture may be more effective than conventional drugs for abdominal pain relief of patients with irritable bowel syndrome. ${ }^{17}$ However, although several studies have shown that acupuncture can relieve $\mathrm{BC},{ }^{18-21}$ the immediate analgesia of acupuncture for BC still needs to be verified.

In the peripheral system, acupuncture can inhibit the contractions of Oddi's sphincter, bidirectionally regulate the gallbladder pressure, promote the secretion of hepatic bile and so on. ${ }^{22-25}$ However, the central mechanism underlying acupuncture for BC relief still needs further investigation. Brain activity is closely correlated with the regulation of visceral function, and central integration is the key to achieving good regulation of visceral function by acupuncture. ${ }^{26}{ }^{27}$ At the same time, the state of acupoints and the function of the viscera are considered to be dynamic, having a two-way relationship with one another. ${ }^{28}$

The rapid development of neuroimaging technologies, such as functional MRI (fMRI), positron emission tomography, electroencephalography, functional nearinfrared spectroscopy (fNIRS), has provided possibilities for neural activity research. Among them, fMRI is the most commonly used technique. However, in the current study, it is difficult for patients with BC to remain confined during MRI, with their body and head motions strictly controlled. ${ }^{29}$ Compared with fMRI, fNIRS is similarly successful in detecting brain functional activities by measuring concentration changes of oxygenated and deoxygenated haemoglobin $(\triangle \mathrm{HbO}$ and $\triangle \mathrm{HbR})$, but it is more flexible without these limitations and has a better temporal resolution. ${ }^{30}$ In addition, fNIRS is also a noninvasive method and has better spatial resolution than electroencephalography. ${ }^{31}$ This neuroimaging technique has been used to assess cortical activity in diverse diseases, such as pain disease, ${ }^{32}$ attention-deficit/hyperactivity disorder, ${ }^{33}$ cerebral palsy ${ }^{34}$ and cognitive impairment. ${ }^{35}$

The current study aims to (1) retestify the immediate analgesic effect of acupuncture by comparing it to sham acupuncture; (2) investigate the potential central mechanism of this analgesic effect by using fNIRS to monitor patients' brain activity during acupuncture procedure; (3) explore the underlying peripheral-cerebral influence by correlating the clinical improvement and pain threshold of acupoint with cerebral neural activity.

\section{METHODS}

\section{Study design}

This is a 1:1 randomised placebo-controlled trial with two parallel groups. This trial will be reported in accordance with the Standard Protocol Items: Recommendations for Intervention Trials guidelines ${ }^{36}$ and will follow the principles of the Consolidated Standards of Reporting Trials and Standards for Reporting Interventions in Clinical Trials of Acupuncture statements. ${ }^{3738}$
Seventy-two participants will be recruited in this study, with 36 participants allotted to each group. All participants will receive one session of acupuncture treatment. As shown in figures 1 and 2, clinical measurements and behavioural data of all participants will be assessed before and after their acupuncture treatment. Each patient's cerebral activity will be synchronously detected by fNIRS during acupuncture treatment.

\section{Patient population and recruitment strategy}

Participants will be screened and recruited from the emergency department and the general surgery department of the First Teaching Hospital of Chengdu University of Traditional Chinese Medicine (TCM) (Chengdu, China) and the First People's Hospital of Longquanyi District (Chengdu, China) from July 2020 to December 2021. Trained researchers will conduct screening of participants. All patients will be fully informed of the experimental procedures, and informed consent will be obtained from all participants prior to their enrolment. Participants will be informed that they are free to withdraw from the study without penalty at any time. For this study, the diagnosis of gallbladder stone disease (GSD) follows the criteria of the European Association for the Study of the Liver. ${ }^{39}$ Patients with GSD who manifest BC and match the inclusion criteria will be included.

\section{Inclusion criteria}

The study inclusion criteria are as follows:

1. Age between 18 and 60 years.

2. Right handed.

3. Mild tenderness in the right upper abdomen, with Murphy's sign (-).

4. No history of having taken any NSAIDs within 2 weeks before enrolment.

5. Signed the informed consent form.

\section{Exclusion criteria}

The study exclusion criteria are as follows:

1. Calculus in the bile duct.

2. Acute cholecystitis, acute suppurative, gangrenous, incarcerated cholecystitis, or perforation of the gallbladder with diffuse peritonitis.

3. Serious complications or primary diseases affecting the cerebral, cardiovascular, liver, kidney, endocrine or haematopoietic system.

4. Severe digestive system diseases, such as peptic ulcer, upper gastrointestinal haemorrhage, gastric tumour, Crohn's disease or irritable bowel syndrome.

5. Other causes of upper abdominal pain, such as gastritis, enteritis, pancreatitis or peritonitis.

6. White cell count of greater than $15 \times 10^{9} / \mathrm{L}$ with a systemic inflammatory response.

7. Mental disorders, such as serious anxiety and/or depression (at least 70 points on the Zung Self-Rating Anxiety Scale or at least 72 points on the Zung Selfrating Depression Scale). ${ }^{40} 41$ 


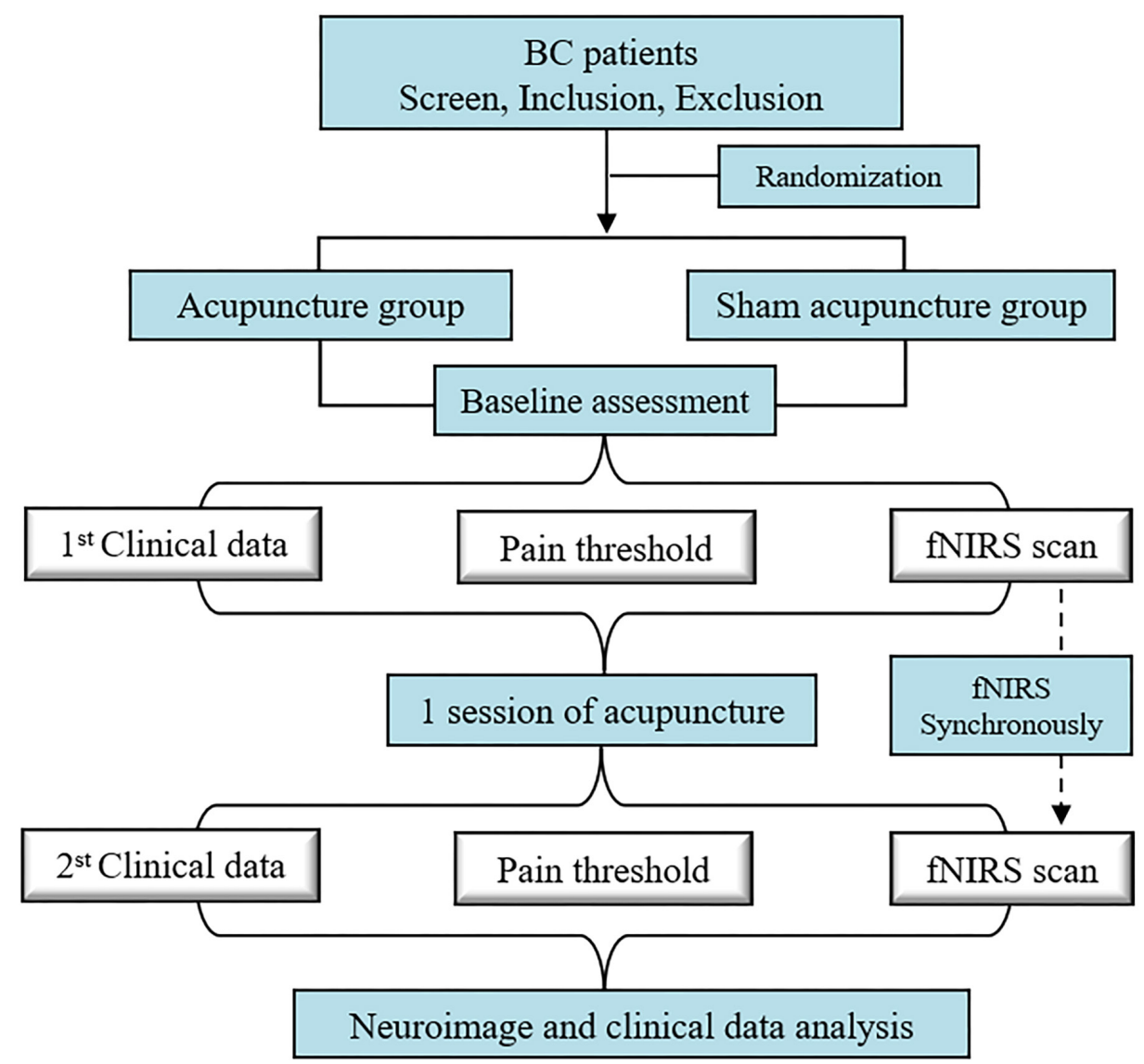

Figure 1 Flow chart of the study design. BC, biliary colic; fNIRS, functional near-infrared spectroscopy.

8. Taking other medications, such as chronic analgesic drugs.

9. Pregnancy or lactating women.

10. History of participation in any other clinical trial in the past 3 months.

11. History of receiving any acupuncture treatment in the past month.

\section{Sample size considerations}

According to a previous study, a Visual Analog Scale score of acute pain decreased by 2.4 points after acupuncture and by 0.7 points after sham acupuncture. ${ }^{42}$ In this study, we anticipate a reduction in the Numeric Rating Scale (NRS) score by 2.4 points after acupuncture and a reduction by one point after sham acupuncture. With $\alpha=0.05$, $1-\beta=0.8$ and a SD of 2, 33 participants would be required per group. Estimating a dropout rate of $10 \%$, we planned to enrol 72 participants, with 36 allotted to each group. Based on previous neuroimaging studies, the optimal sample size for a study is between 16 and 32 subjects. ${ }^{43}$ Considering the dropout rate and loss of data due to head motion, 25 participants per group will undergo fNIRS imaging in this trial.

\section{Randomisation and blinding}

A random sequence will be generated by a third-party professional statistician using the SAS V.9.2 software (SAS Institute). The randomisation list will be placed in sealed opaque envelopes and opened by a non-involved investigator in consecutive order. A total of 72 participants with GSD will be randomised in a ratio of 1:1 to the acupuncture with Yanglingquan (GB34) group or the sham acupuncture group, with 36 patients in each group. It is difficult to blind the acupuncturists to treatment allocation, but the patients, outcome assessors, and statisticians will be blinded to treatment allocation. Participants in different groups will be separated into cubicles to limit communication, and the same type of Park device will be used for ensuring the blinding of participants. Moreover, participants will be told that there are two different types of acupuncture provided in this study; terms such as "placebo" or "sham" will not be used. Therefore, sham acupuncture aims to control not only the superficial and cutaneous somatosensory effects around the GB34 acupoint but also the cognitive processing induced by the participants' expectations of acupuncture. ${ }^{44}$

\section{Patient and public involvement}

Patients in this trial will not be involved in the design, recruitment or conduct of the study. The patients will be able to view the study results via social media.

\section{Interventions}

Participants will receive one session of acupuncture either on the right GB34 or on the sham acupoint, which will be conducted by trained, licensed acupuncturists with at 


\begin{tabular}{|c|c|c|c|c|}
\hline \multicolumn{5}{|c|}{ STUDY PERIOD } \\
\hline Items & \multicolumn{2}{|c|}{ Baseline } & \multirow{2}{*}{$\begin{array}{c}\begin{array}{c}\text { Acupuncture } \\
\text { (one session) }\end{array} \\
\text { 1-week }\end{array}$} & \multirow{2}{*}{$\begin{array}{c}\begin{array}{c}\text { Outcome } \\
\text { assessment }\end{array} \\
\text { 1-week } \\
\end{array}$} \\
\hline TIMEPOINT & 0 -week & 0 -week & & \\
\hline \multicolumn{5}{|l|}{ ENROLMENT: } \\
\hline \multirow{4}{*}{$\begin{array}{l}\text { Eligibility screen } \\
\text { Informed consent } \\
\text { Physical examination } \\
\text { Allocation }\end{array}$} & $x$ & & & \\
\hline & $x$ & & & \\
\hline & $x$ & & & \\
\hline & & $x$ & & \\
\hline \multicolumn{5}{|l|}{ INTERVENTIONS: } \\
\hline \multirow{2}{*}{$\begin{array}{l}\text { Verum acupuncture } \\
\text { Sham acupuncture }\end{array}$} & & & $x$ & $x$ \\
\hline & & & $x$ & $x$ \\
\hline \multicolumn{5}{|l|}{ ASSESSMENTS: } \\
\hline \multirow{2}{*}{$\begin{array}{l}\text { NRS } \\
\text { fNIRS }\end{array}$} & & & $x$ & \\
\hline & & $x$ & & $x$ \\
\hline Pain threshold & $x$ & & & \\
\hline SAS, SDS & $x$ & & & \\
\hline \multirow[b]{2}{*}{ Needle sensation } & & & $x$ & \\
\hline & & $x$ & & $x$ \\
\hline \multicolumn{5}{|c|}{ PARTICIPANTS SAFETY: } \\
\hline Adverse events & & & $x$ & \\
\hline
\end{tabular}

Figure 2 Standard protocol items. fNIRS, functional near-infrared spectroscopy; NRS, Numeric Rating Scale; SAS, Zung Selfrating Anxiety Scale; SDS, Zung Self-rating Depression Scale; SF-12, 12-item Short-form Health Survey.

least 3 years of clinical experience in acupuncture. The sham acupoint is on the lateral side of the tibia, 1 to $2 \mathrm{~cm}$ lateral to ST36 (Zusanli) ${ }^{45}$ (figure 3). The location of GB34 is on the lateral side of the lower leg, in the depression anterior and inferior to the head of the fibula. The location of ST36 is four fingers' breadth below the lower margin of the patella and one finger's breadth laterally from the anterior crest of the tibia.
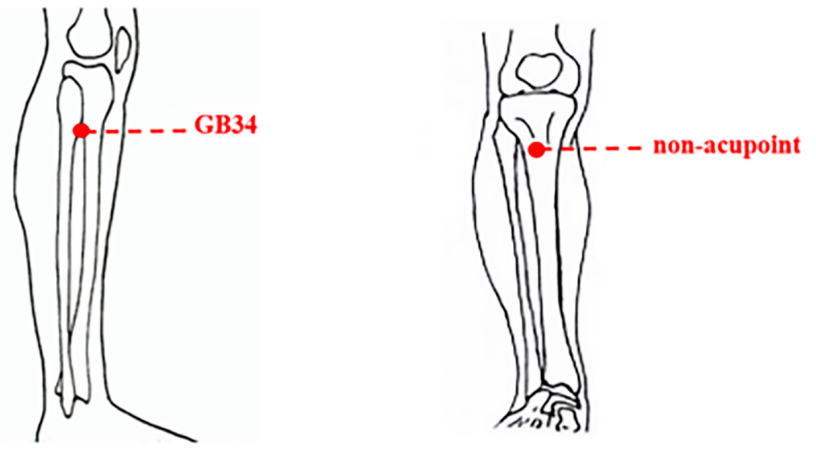

Figure 3 Location of acupoint or non-acupoint in the trial.
Both groups will undergo the same acupuncture procedure and manipulation. For the acupuncture procedure, four different acupuncture manipulations will be used. Specifically, the first task is needle insertion (NI), followed by an initial twirl to activate the acupoint. The second task is the first run of needle twirl, which is repeated four times (T1, T2, T3, and T4) to keep the acupoint activated. The duration of the second task is $140 \mathrm{~s}$, including four twirls $(4 \times 20 \mathrm{~s})$ and three rest times $(3 \times 20 \mathrm{~s})$. Then, the needle remains for $5 \mathrm{~min}$ to maintain deqi sensation. The third task is the second run of needle twirl, which is repeated four times (T5, T6, T7, and T8) to strengthen the deqi sensation. The third task is the same length of time as the second task (140s). Finally, the last task is needle removal (NR). The acupuncture procedure will last a total of $10 \mathrm{~min}$ and $30 \mathrm{~s}$ (figure 4).

For acupuncture manipulation, disposable sterile filiform needles $(0.25 \times 40 \mathrm{~mm}$; Huatuo, Suzhou, China) with a Park device will be used in the acupuncture group to be perpendicularly inserted into the point at a depth of 21-26 mm after skin disinfection using alcohol (figure 5). During each needle twirl, the needle will be 


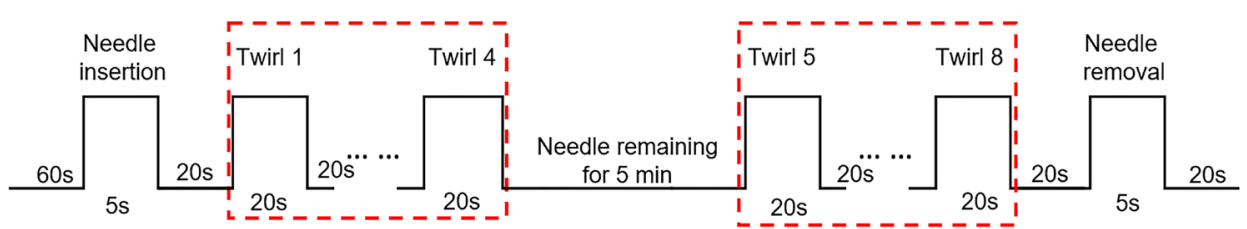

Figure 4 Experimental paradigm.

bidirectionally twisted within $90^{\circ}$ to $180^{\circ}$, lifted, and thrust at an even amplitude between 3 and $5 \mathrm{~mm}$ for 1 to $1.5 \mathrm{~Hz}$ (60-90 times/min) to induce and maintain the deqi sensation (including soreness, numbness, distention, and heaviness). ${ }^{46} 47$

The sham acupuncture group will receive non-insertive acupuncture using the Park sham needle supported by the Park device (figure 5). ${ }^{48}$ The acupuncturist will be instructed to lightly position the sham needle against the surface of the skin to minimise any physiological effect. ${ }^{49}$

Acupuncture will be discontinued and patients will be treated immediately when they experience severe discomforts, such as pain, bleeding or fainting. After finishing the acupuncture treatment, participants will be able to receive medication if they still have $\mathrm{BC}$, and the type and dosage of medication used will also be recorded in the case report form (CRF).

\section{Outcome measures}

\section{Clinical outcome assessments}

The primary outcome is the mean difference in the change in pain intensity between before and after an intervention. To measure patients' self-reported pain intensity before and after acupuncture, ${ }^{50} 51$ the NRS will be used. The NRS is a horizontal line with 10 segments labelled from 0 ('no pain at all') to 10 ('the worst pain imaginable').

Other clinical data, including demographic information, participants' quality of life, and psychosocial state at the baseline, will also be recorded. The standard protocol items of the study are exhibited in figure 2. The 12-item Short-form Health Survey, ${ }^{52}$ Zung Self-rating Anxiety Scale $^{40}$ and Zung Self-rating Depression Scale ${ }^{41}$ will be used to evaluate participants' quality of life and psychosocial state at baseline.

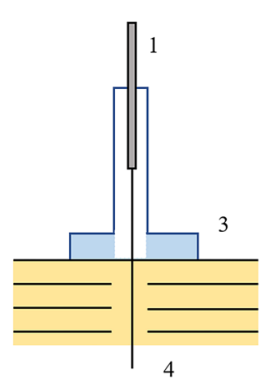

Verum acupuncture

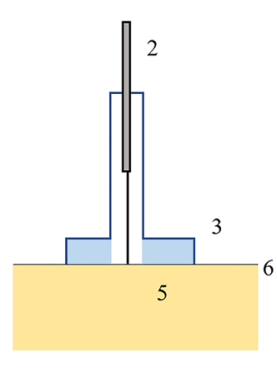

Sham acupuncture
Real needle

Retractable needle shaft Park device

Dull tip of placebo needle Skin
Sharp tip of real needle

Figure 5 Verum acupuncture and sham acupuncture.
To assess deqi during acupuncture, a needle-sensation evaluation form will be deployed to two groups at four time points: after NI, after T4, after T8, and after NR. This form is based on the Chinese version of the modified Massachusetts General Hospital Acupuncture Sensation Scale (C-MASS), and its validity and reliability have been previously examined. ${ }^{53}$ Needle sensation is an indispensable part of acupuncture treatment. ${ }^{55}$ The needlesensation evaluation form includes deqifeatures (soreness, distention, numbness, heaviness, dull pain, and so on) and a rating of deqi intensity. Patients will be asked to describe the intensity of deqi with a 10-point Visual Analog Scale labelled from 0 ('none') to 10 ('unbearable').

\section{Pain threshold measurements of the acupoint}

Based on the TCM theory, a disorder of the viscera inside the body must have some manifestation outside, and the acupoint can show a person's response to disease externally. To explore the activation of the (sham) acupoint and its association with pain relief among patients with BC after acupuncture, the mechanical and pressure pain thresholds (PPTs) will be measured.

The mechanical pain threshold will be measured using the electronic Von Frey esthesiometer (model 2390; IITC Life Science, Woodland Hills, California, USA). It has been found that this device can detect hyperalgesia and changes in sensory nerve function. ${ }^{56-58}$ At the point, a progressive pressure will be applied at a rate of $10 \mathrm{~g} / \mathrm{s}$. The average mechanical pain threshold for the GB34 point/sham acupoint will be calculated.

The PPT will be measured using the FDIX force gauge (Force One FDIX; Wagner Instruments, Greenwich, Connecticut, USA). PPT is widely used in clinical practice as a semiobjective method with which to quantify localised pain. ${ }^{5960}$ At the point, a progressive pressure will be applied at a rate of $100 \mathrm{gf} / \mathrm{s}$. The average PPT for the GB34 point/sham acupoint will be calculated.

If there is a difference of more than $15 \mathrm{~g} / 500 \mathrm{gf}$ (separately for the mechanical pain threshold and PPT) between the two measurements performed at one acupoint, a third group of measurements will be taken. The two devices described have been used in our previous study. ${ }^{61}$

fNIRS imaging and data collection fNIRS - a method of optical neuroimaging that characterises haemodynamic changes at the brain's surface in a priori-identified regions of the brain-will be used for the measurement of cortical neural activation of patients' brains. An fNIRS system (OMM-3000; Shimadzu, Kyoto, Japan) that has 
continuous-wave laser diodes with 780,805 and $830 \mathrm{~nm}$ wavelengths will be used in this experiment. ${ }^{62}$ The optodes will be positioned over the international 10-20 system for electroencephalography electrode placement by using individually sized caps selected according to head circumference. About 10 locations will be spatially adjusted across all cap sizes to maintain consistent coverage of our regions of interest. ${ }^{6364}$ For accurate fixation, the optodes will be mounted on the holder in the NIRS cap, and the distance between pairs of light sources and detector optodes will be set to $2.5-3 \mathrm{~cm} .{ }^{65}$

In the present study, eight emitters and eight detectors will be used to thoroughly investigate the dorsolateral prefrontal cortex (DLPFC) and primary somatosensory cortex of the human brain. The emitters will be positioned by considering $\mathrm{Fpz}$ and $\mathrm{Cz}$ as reference points. A total of 20 channels will be configured using emitterdetector combinations.

\section{Safety and monitoring}

To provide an overview of their health status, each participant in this study will undergo a series of health evaluations, including an abdominal ultrasound, blood biochemical test and ECG examination. Any acupuncture treatment-related adverse events, such as haematoma, subcutaneous haemorrhage, serious pain, local infection, broken needle and fatigue, will be processed immediately and recorded in detail in the CRF. Adverse events of the acupuncture group and sham acupuncture group will be compared.

\section{Quality control and data collection and management}

All researchers will receive a series of training before the start of this study, to ensure that they fully understand the study protocol and standard procedures. CRFs labelled by unique numeric identifiers will be used to collect relevant data, adverse events and safety assessments for patients. Only outcome assessors have access to CRFs and will perform the double data entry. The Evidence-based Medicine Center of the Chengdu University of TCM will be in charge of supervising the study and monitoring data every 3 months.

\section{STATISTICAL ANALYSIS \\ Clinical data analysis}

Clinical data will be statistically analysed using the Statistical Package for the Social Sciences V.22.0 software program (IBM Corporation), with a significance level of 0.05 , and all hypothesis tests will be two tailed. Skewed data distribution will be normalised. Means, SD and 95\% CIs will be computed to describe continuous variables.

Baseline demographic characteristics, NRS scores and pain threshold values between the two groups will be analysed with the $\chi^{2}$ test or Fisher's exact test for categorical measures and with the t-test or Wilcoxon rank-sum test for continuous measures. The needle sensation will be compared between the two groups at four time points by using a $2 \times 4$ repeated-measures analysis of covariance approach.

\section{fNIRS data analysis}

Data will be analysed in MATLAB V.8.6 (Mathworks, Natick, Massachusetts, USA). First, a wavelet motioncorrection procedure will be used for motion artefacts with all-optical density data ${ }^{64} \mathrm{Next}$, the optical density data will be converted to $\mathrm{HbO}$ and $\mathrm{HbR}$ values using the modified Beer-Lambert law. ${ }^{66}$ Data from individual channels will be visually inspected for excessive noise. Channels with excessive noise will be excluded from subsequent analyses. In addition, we will employ a wavelet-based method to remove global noise, which is used as a powerful mathematical tool in fNIRS data preprocessing. ${ }^{67-69}$ To obtain an average response for each of the 20 channels in each participant, the haemodynamic response function will be estimated by a general linear model approach using ordinary least squares.

Correlation analyses will be used to investigate associations among clinical outcomes, pain threshold values of the acupoint and fNIRS data. Differences in correlations between two groups will be further examined by grouplevel analysis.

\section{DISCUSSION}

This is a randomised controlled, paralleled clinical trial seeking to explore the immediate pain alleviation of acupuncture for $\mathrm{BC}$ and its influence on the peripheral sensitised acupoint and central brain activity.

According to the TCM theory, GB34 is the Lower He-sea point of the gallbladder and is considered specifically connected to the gallbladder. As one of the most common acupoints used for GSD treatment, acupuncture with GB34 is considered effective for the treatment of gallstone disease. ${ }^{192024}$ Some clinical studies have reported that sham acupuncture interventions, such as needle insertion at a sham acupoint, are not entirely ineffective due to their associations with moderately large, non-specific effects. ${ }^{70} 71$ Thus, in clinical trials, sham acupuncture involving penetrating needling is not recommended. ${ }^{72}{ }^{73}$ To better verify the effectiveness of manual acupuncture with GB34, a non-inserted needle at a non-acupoint is chosen as the sham control technique to minimise physiological effects in the sham group. Thus, a non-acupoint on the lateral side of the lower leg, which is almost at the same level as the GB34 acupoint, will be chosen in the sham group to blind participants in this study. However, a recent review indicates that sham acupuncture and verum acupuncture have almost identical efficacy when the dermatomes that are stimulated highly overlap. ${ }^{74}$ The present study may help to test these authors' arguments. Because the pain of patients with $\mathrm{BC}$ is so severe that they can tolerate fNIRS imaging for only a short time, acupuncture with a short-time needle retention time ( $10 \mathrm{~min}$ and $30 \mathrm{~s}$ ) will be chosen after the pilot study. 
Recent Chinese research has found that, when the body is in a state of disease, disease-related acupoints can be sensitised. ${ }^{75-77}$ Pain sensitivity is one of the most important sensitivity manifestations at sensitised acupoints, and its degree may be correlated with disease condition. ${ }^{57}{ }^{58}$ Considering the importance of acupoint sensitisation for the acupuncture effect, we will measure the pain threshold of GB34 and observe the efficacy of acupuncture at GB34 for the treatment of BC. In addition to NRS and C-MASS, which offer subjective-state measurements, we also evaluated the impact of acupuncture treatment on objective outcomes using pain threshold values and the fNIRS data. Meanwhile, correlations will be analysed to investigate associations among clinical outcomes, pain threshold values of the acupoint, and fNIRS data.

fNIRS is suitable for all possible participant populations, from newborns to the elderly, and in a variety of experimental settings both inside and outside the laboratory ${ }^{7879}$ Some diseases with acute pain, such as BC, are difficult to investigate in tightly restrained environments, such as an fMRI system. The process of fNIRS recording is silent, relatively comfortable and does not impose physical constraints on the participant, ${ }^{80}$ which means it is a good choice of imaging modality for many studies of patients with acute pain. Moreover, a major reason for choosing fNIRS is that the usage of metal objects is applicable. For acupuncture studies, because acupuncture needles are magnetic metals, it is impossible to implement treatment and fMRI imaging at the same time. Therefore, using fNIRS, the synchronisation of acupuncture treatment and brain-image collection can be realised.

According to previous literature, acupuncture stimulation modulates the cortical activities of the somatomotor area in humans. ${ }^{81} 82$ Moreover, acupuncture at GB34 can activate the somatomotor area and DLPFC in healthy subjects in our pilot trial. The DLPFC has displayed a critical role in pain modulation. ${ }^{83}$ In particular, DLPFC is a key node of several brain networks and may be a key node of networks implicated in nociceptive processing and control. ${ }^{84} \mathrm{~A}$ study reported that left DLPFC activity is negatively correlated with pain unpleasantness. ${ }^{85}$ Freund et al found that subjects given instructions to suppress pain show increased activation of the bilateral DLPFC during prolonged acute pain stimulation. ${ }^{86}$ There is converging evidence that the DLPFC has a role in cognitive components of the pain experience. Therefore, the somatomotor area and DLPFC will be chosen as brain regions of interest for this study.

In this trial, the following measures will be taken to strengthen quality control: (1) this study restricts participants to ages between 18 and 60 years and to being right handed, avoiding the impact of age and handedness on brain function and structure; (2) to get stable statistic power, 36 patients will be included in each group for clinical evaluation and 25 patients in each group for the central mechanism study; (3) all researchers will be uniformly trained to understand the design and process of the trial, and the acupuncture treatment will be performed by two licensed acupuncturists, who have received special and standardised training before the trial; (4) one technician will perform all scans in the same fNIRS machine according to the operation standard and the design of the study in the fixed condition, and, before being scanned, the mental state of each participant will be evaluated using the Zung Self-rating Anxiety Scale and Zung Self-rating Depression Scale.

In summary, this study is planned as a randomised controlled, paralleled clinical trial to evaluate the efficacy and central mechanism of acupuncture treatment on BC compared with sham acupuncture. To our knowledge, it is the first functional brain imaging study focusing on exploring the immediate pain alleviation of acupuncture for BC and its influence on the peripheral sensitised acupoint and central brain activity. The findings may provide evidence of the clinical benefits and mechanisms of acupuncture for patients with BC.

Contributors RS, F-rL and NS participated in the conception and design of the trial. NS, D-MH and XY participated in drafting the manuscript. LB, XD, YZ, T-HZ and SS will be responsible for participants recruitment. NS and $Y Z$ will perform the acupuncture for participants. ZL, SC and JC will be in charge of data collection. NS, RS and F-JZ will conduct data analysis. All authors discussed, read, revised the manuscript and all approved the publication of this protocol.

Competing interests None declared.

Patient and public involvement Patients and/or the public were not involved in the design, or conduct, or reporting, or dissemination plans of this research.

Patient consent for publication Not applicable.

Ethics approval This study was approved by Sichuan Regional Ethics Review Committee of Traditional Chinese Medicine (2019KL-029); Medical Ethics Committee of Hospital Affiliated to Chengdu University of Traditional Chinese Medicine (AF-KY-2020071). Participants gave informed consent to participate in the study before taking part.

Provenance and peer review Not commissioned; externally peer reviewed.

Open access This is an open access article distributed in accordance with the Creative Commons Attribution Non Commercial (CC BY-NC 4.0) license, which permits others to distribute, remix, adapt, build upon this work non-commercially, and license their derivative works on different terms, provided the original work is properly cited, appropriate credit is given, any changes made indicated, and the use is non-commercial. See: http://creativecommons.org/licenses/by-nc/4.0/.

\section{ORCID iDs}

Ning Sun http://orcid.org/0000-0002-1064-5705

Yuanfang Zhou http://orcid.org/0000-0002-5848-6432

Yuzhu Qu http://orcid.org/0000-0002-1221-9427

Shirui Cheng http://orcid.org/0000-0001-7866-119X

Ruirui Sun http://orcid.org/0000-0003-0555-2042

Fan-rong Liang http://orcid.org/0000-0001-8518-9268

\section{REFERENCES}

1 Johnston MJ, Fitzgerald JEF, Bhangu A, et al. Outpatient management of biliary colic: a prospective observational study of prescribing habits and analgesia effectiveness. Int J Surg 2014;12:169-76.

2 The epidemiology of gallstone disease in Rome, Italy. Part II. factors associated with the disease. The Rome group for epidemiology and prevention of cholelithiasis (GREPCO). Hepatology 1988;8:907-13.

3 Baiu I, Hawn MT. Gallstones and biliary colic. JAMA 2018;320:1612.

4 Portincasa P, Moschetta A, Petruzzelli M, et al. Gallstone disease: symptoms and diagnosis of gallbladder stones. Best Pract Res Clin Gastroenterol 2006;20:1017-29.

5 Ansaloni L, Pisano M, Coccolini F. WSES guidelines on acute calculous cholecystitis. World J Emerg Surg 2016;2016:25. 
6 Attili AF, De Santis A, Capri R, et al. The natural history of gallstones: the GREPCO experience. The GREPCO group. Hepatology 1995;21:656-60.

7 Everhart JE, Ruhl CE. Burden of digestive diseases in the United States Part III: liver, biliary tract, and pancreas. Gastroenterology 2009;136:1134-44.

8 Shaffer EA. Epidemiology and risk factors for gallstone disease: has the paradigm changed in the 21 st century? Curr Gastroenterol Rep 2005; 7:132-40.

9 Fraquelli M, Casazza G, Conte D, et al. Non-Steroid antiinflammatory drugs for biliary colic. Cochrane Database Syst Rev 2016;9:CD006390.

10 García-Rayado G, Navarro M, Lanas A. Nsaid induced gastrointestinal damage and designing Gl-sparing NSAIDs. Expert Rev Clin Pharmacol 2018;11:1031-43.

11 Aminoshariae A, Kulild JC, Donaldson M. Short-Term use of nonsteroidal anti-inflammatory drugs and adverse effects: an updated systematic review. J Am Dent Assoc 2016;147:98-110.

12 Long B, April MD. Are nonsteroidal anti-inflammatory drugs efficacious in relieving biliary colic pain? Ann Emerg Med 2017;70:822-4.

13 Beltaief K, Grissa MH, Msolli MA, et al. Acupuncture versus titrated morphine in acute renal colic: a randomized controlled trial. J Pain Res 2018;11:335-41.

14 Grissa MH, Baccouche H, Boubaker $\mathrm{H}$, et al. Acupuncture vs intravenous morphine in the management of acute pain in the ED. Am J Emerg Med 2016;34:2112-6.

15 Kaynar M, Koyuncu F, Buldu İbrahim, et al. Comparison of the efficacy of diclofenac, acupuncture, and acetaminophen in the treatment of renal colic. Am J Emerg Med 2015;33:749-53.

16 Pei L, Geng H, Guo J, et al. Effect of acupuncture in patients with irritable bowel syndrome: a randomized controlled trial. Mayo Clin Proc 2020;95:1671-83.

17 Zhao L, Li D, Zheng $\mathrm{H}$, et al. Acupuncture as adjunctive therapy for chronic stable angina: a randomized clinical trial. JAMA Intern Med 2019;179:1388-97.

18 Jiang HF, Zhao C, Tang XF. Clinical analgesic effect of acupuncture at GB34 (Yanglingquan) and EX-LE6 (Dannang) for biliary colic caused by acute cholecystitis. J Hepatopancreatobiliary Surg 2016;28:481-3.

19 Cao ZY, Zhang YB, Zhang HC. Effects of electroacupuncturing at Yanglingquan on volume and arterial hemodynamics of gallbladder in chronic cholecystitis. Chinese Journal of Basic Medicine in Traditional Chinese Medicine 2016;22:1228-31.

20 Chen WH, HW Y. Puncturing on Yanglingquan point for relieving of colic pain of bile cyst. Acupunct Res 2000;01:62-3.

21 CB L, Zhu JM, Huang JP. Pain-Relieving effects of acupuncture at Yanglingquan and Dannang acupoints in acute cholecystitis. Shanghai Journal of Traditional Chinese Medicine 2011;45:56-7.

$22 \mathrm{Kim} \mathrm{MH}$. A brief commentary: electroacupuncture may relax the contraction of sphincter of Oddi. J Altern Complement Med 2001;7 Suppl 1:119-20.

23 Lee SK, Kim MH, Kim HJ, et al. Electroacupuncture may relax the sphincter of Oddi in humans. Gastrointest Endosc 2001;53:211-6.

24 Zhao J, Yu Y, Luo M, et al. Bi-Directional regulation of acupuncture on extrahepatic biliary system: an approach in guinea pigs. Sci Rep 2017;7:14066.

$25 \mathrm{Ma} \mathrm{C}$, Yang W. [The preventing and treating effects of electroacupuncture on cholelithiasis in golden hamster]. Zhen Ci Yan Jiu 1996;21:68-72

26 Catani M, Dell'acqua F, Thiebaut de Schotten M. A revised limbic system model for memory, emotion and behaviour. Neurosci Biobehav Rev 2013;37:1724-37.

27 Zhang F, Wu L, Zhao J, et al. Neurobiological mechanism of acupuncture for relieving visceral pain of gastrointestinal origin. Gastroenterol Res Pract 2017;2017:5687496

28 Liang C, Wang K, Xu B, et al. Electroacupuncture at acupoint ST 37(Shangjuxu) improves function of the enteric nervous system in a novel mouse constipation model. BMC Complement Altern Med 2016;16:392.

29 Lu W, Dong K, Cui D, et al. Quality assurance of human functional magnetic resonance imaging: a literature review. Quant Imaging Med Surg 2019;9:1147-62.

30 Naseer N, Hong K-S. fNIRS-based brain-computer interfaces: a review. Front Hum Neurosci 2015;9:3.

31 Huppert TJ, Diamond SG, Franceschini MA, et al. Homer: a review of time-series analysis methods for near-infrared spectroscopy of the brain. Appl Opt 2009;48:D280-98.

32 Fernandez Rojas R, Huang X, Ou K-L. Toward a functional nearinfrared spectroscopy-based monitoring of pain assessment for nonverbal patients. J Biomed Opt 2017;22:1-12.
$33 \mathrm{Gu}$ Y, Miao S, Han J, et al. Complexity analysis of fNIRS signals in ADHD children during working memory task. Sci Rep 2017;7:829.

34 Sukal-Moulton T, de Campos AC, Alter KE, et al. Relationship between sensorimotor cortical activation as assessed by functional near infrared spectroscopy and lower extremity motor coordination in bilateral cerebral palsy. Neuroimage Clin 2018;20:275-85.

35 Yoon JA, Kong IJ, Choi J, et al. Neural compensatory response during complex cognitive function tasks in mild cognitive impairment: a near-infrared spectroscopy study. Neural Plast 2019;2019:7845104

36 Chan A-W, Tetzlaff JM, Gøtzsche PC, et al. Spirit 2013 explanation and elaboration: guidance for protocols of clinical trials. $B M J$ 2013;346:e7586.

37 Schulz KF, Altman DG, Moher D, et al. Consort 2010 statement: updated guidelines for reporting parallel group randomised trials. BMJ 2010;340:c332.

38 MacPherson $\mathrm{H}$, Altman DG, Hammerschlag R, et al. Revised standards for reporting interventions in clinical trials of acupuncture (stricta): extending the CONSORT statement. PLOS Med 2010;7:e1000261.

39 European Association for the Study of the Liver (EASL). Electronic address: easloffice@easloffice.eu. EASL clinical practice guidelines on the prevention, diagnosis and treatment of gallstones. $J$ Hepatol 2016:65:146-81.

40 Zung WW. A rating instrument for anxiety disorders. Psychosomatics 1971;12:371-9.

41 Zung WW. A self-rating depression scale. Arch Gen Psychiatry 1965;12:63-70.

42 Wang L-P, Zhang X-Z, Guo J, et al. Efficacy of acupuncture for acute migraine attack: a multicenter single blinded, randomized controlled trial. Pain Med 2012;13:623-30.

43 Friston K. Ten ironic rules for non-statistical reviewers. Neuroimage 2012;61:1300-10.

44 Dhond RP, Yeh C, Park K, et al. Acupuncture modulates resting state connectivity in default and sensorimotor brain networks. Pain 2008;136:407-18.

45 Zeng F, Qin W, Ma T, et al. Influence of acupuncture treatment on cerebral activity in functional dyspepsia patients and its relationship with efficacy. Am J Gastroenterol 2012;107:1236-47.

46 Yin T, He Z, Ma P, et al. Effect and cerebral mechanism of acupuncture treatment for functional constipation: study protocol for a randomized controlled clinical trial. Trials 2019;20:283.

47 Sun R, Hong X, Guo J, et al. The central mechanism of acupuncture treatment with a long-lasting effect for functional dyspepsia: study protocol for a randomized controlled trial. Trials 2018;19:373.

48 Park J, White A, Stevinson C, et al. Validating a new non-penetrating sham acupuncture device: two randomised controlled trials. Acupunct Med 2002;20:168-74.

49 Lund I, Lundeberg T. Are minimal, superficial or sham acupuncture procedures acceptable as inert placebo controls? Acupunct Med 2006;24:13-15.

50 Farrar JT, Young JP, LaMoreaux L, et al. Clinical importance of changes in chronic pain intensity measured on an 11-point numerical pain rating scale. Pain 2001;94:149-58.

51 Ferreira-Valente MA, Pais-Ribeiro JL, Jensen MP. Validity of four pain intensity rating scales. Pain 2011;152:2399-404

52 Ware J, Kosinski M, Keller SD. A 12-Item short-form health survey: construction of scales and preliminary tests of reliability and validity. Med Care 1996;34:220-33.

53 Kong J, Gollub R, Huang T, et al. Acupuncture de Qi, from qualitative history to quantitative measurement. J Altern Complement Med 2007:13:1059-70.

54 Yu DTW, Jones AYM, Pang MYC. Development and validation of the Chinese version of the Massachusetts General Hospital acupuncture sensation scale: an exploratory and methodological study. Acupunct Med 2012;30:214-21.

55 Yuan H-W, Ma L-X, Qi D-D, et al. The historical development of deqi concept from classics of traditional Chinese medicine to modern research: exploitation of the connotation of deqi in Chinese medicine. Evid Based Complement Alternat Med 2013;2013:1-5.

56 Chen Y, Yao Y, Wu Y, et al. Transcutaneous electric acupoint stimulation alleviates remifentanil-induced hyperalgesia in patients undergoing thyroidectomy: a randomized controlled trial. Int J Clin Exp Med 2015;8:5781-7.

57 Neto MLP, Maciel LYS, Cruz KML, et al. Does electrode placement influence tens-induced antihyperalgesia in experimental inflammatory pain model? Braz J Phys Ther 2017:21:92-9.

58 Yamamoto S, Ono H, Kume K, et al. Oxaliplatin treatment changes the function of sensory nerves in rats. $J$ Pharmacol Sci 2016:130:189-93. 
59 Persson AL, Brogårdh C, Sjölund BH. Tender or not tender: testretest repeatability of pressure pain thresholds in the trapezius and deltoid muscles of healthy women. $J$ Rehabil Med 2004;36:17-27.

60 Fischer AA. Pressure algometry over normal muscles. standard values, validity and reproducibility of pressure threshold. Pain 1987;30:115-26.

61 Sun M, Tao S, Geng G, et al. Identification of the optimal points for the acupuncture treatment of neck pain in China: protocol for a multicenter, matched, case-control study. BMJ Open 2019;9:e029194.

62 Scholkmann F, Kleiser S, Metz AJ, et al. A review on continuous wave functional near-infrared spectroscopy and imaging instrumentation and methodology. Neuroimage 2014;85 Pt 1:6-27.

63 Okamoto M, Dan H, Sakamoto K, et al. Three-Dimensional probabilistic anatomical cranio-cerebral correlation via the International 10-20 system oriented for transcranial functional brain mapping. Neuroimage 2004;21:99-111.

64 Tsuzuki D, Cai D-sheng, Dan H, et al. Stable and convenient spatial registration of stand-alone NIRS data through anchor-based probabilistic registration. Neurosci Res 2012;72:163-71.

65 Gratton G, Brumback CR, Gordon BA, et al. Effects of measurement method, wavelength, and source-detector distance on the fast optical signal. Neuroimage 2006;32:1576-90.

66 Molavi B, Dumont GA. Wavelet-based motion artifact removal for functional near-infrared spectroscopy. Physiol Meas 2012;33:259-70.

67 Wyatt JS, Cope M, Delpy DT, et al. Quantification of cerebral oxygenation and haemodynamics in sick newborn infants by near infrared spectrophotometry. Lancet 1986;2:1063-6.

68 Strangman G, Culver JP, Thompson JH, et al. A quantitative comparison of simultaneous BOLD fMRI and NIRS recordings during functional brain activation. Neuroimage 2002;17:719-31.

69 Duan L, Zhao Z, Lin Y, et al. Wavelet-based method for removing global physiological noise in functional near-infrared spectroscopy. Biomed Opt Express 2018:9:3805-20.

70 Linde K, Niemann K, Schneider A, et al. How large are the nonspecific effects of acupuncture? A meta-analysis of randomized controlled trials. BMC Med 2010;8:75.

71 Birch S. A review and analysis of placebo treatments, placebo effects, and placebo controls in trials of medical procedures when sham is not inert. J Altern Complement Med 2006;12:303-10.

72 MacPherson H, Vertosick E, Lewith G, et al. Influence of control group on effect size in trials of acupuncture for chronic pain: a secondary analysis of an individual patient data meta-analysis. PLOS One 2014;9:e93739.
73 Xu S, Yu L, Luo X, et al. Manual acupuncture versus sham acupuncture and usual care for prophylaxis of episodic migraine without aura: multicentre, randomised clinical trial. BMJ 2020;368:m697.

74 Ots T, Kandirian A, Szilagyi I, et al. The selection of dermatomes for sham (placebo) acupuncture points is relevant for the outcome of acupuncture studies: a systematic review of sham (placebo)controlled randomized acupuncture trials. Acupunct Med 2020;38:211-26.

75 Xiao QW, Li J, Ye J. Research on the phenomenon and regularity of acupoint sensitization forcervical spondylosis. China Journal of Traditional Chinese Medicine and Pharmacy 2020;35:89-92.

76 Ye J, Yang H, Xiao QW. Phenomenon and regularity of acupoints sensitization for knee osteoarthritis. China Journal of Traditional Chinese Medicine and Pharmacy 2019;34:5127-30.

77 Wang X, Li J, Ye J. Study on acupoint sensitization of lumbar B disease and its regularity. Lishizhen Medicine and Materia Medica Research 2018;29:1483-6.

78 Ferrari M, Quaresima V. A brief review on the history of human functional near-infrared spectroscopy (fNIRS) development and fields of application. Neuroimage 2012;63:921-35.

79 Pinti P, Tachtsidis I, Hamilton A, et al. The present and future use of functional near-infrared spectroscopy (fNIRS) for cognitive neuroscience. Ann N Y Acad Sci 2020;1464:5-29.

80 Lloyd-Fox S, Blasi A, Elwell CE. Illuminating the developing brain: the past, present and future of functional near infrared spectroscopy. Neurosci Biobehav Rev 2010;34:269-84.

81 Jeun S-S, Kim J-S, Kim B-S, et al. Acupuncture stimulation for motor cortex activities: a 3T fMRI study. Am J Chin Med 2005;33:573-8.

82 Maeda Y, Kim H, Kettner N, et al. Rewiring the primary somatosensory cortex in carpal tunnel syndrome with acupuncture. Brain 2017:140:914-27.

83 Liu J, Chen L, Tu Y, et al. Different exercise modalities relieve pain syndrome in patients with knee osteoarthritis and modulate the dorsolateral prefrontal cortex: a multiple mode MRI study. Brain Behav Immun 2019;82:253-63.

84 Seminowicz DA, Moayedi M. The dorsolateral prefrontal cortex in acute and chronic pain. J Pain 2017;18:1027-35.

85 Lorenz J, Minoshima S, Casey KL. Keeping pain out of mind: the role of the dorsolateral prefrontal cortex in pain modulation. Brain 2003;126:1079-91.

86 Freund W, Klug R, Weber F, et al. Perception and suppression of thermally induced pain: a fMRI study. Somatosens Mot Res 2009;26:1-10. 